\title{
QUANTUM TECHNOLOGIES IMPACT ON ELECTROMAGNETIC SPECTRUM MONITORING
}

\author{
Annamaria SÂRBU \\ paljanosanna@yahoo.com \\ Paul BECHET \\ pbechet@gmail.com \\ Tiberiu GIURGIU \\ giurgiutiberiu@yahoo.com \\ “NICOLAE BăLCESCU”LAND FORCES ACADEMY, SIBIU, ROMANIA

\begin{abstract}
Electromagnetic spectrum (EMS) superiority represents a prerequisite for resilient defence strategies, capable of effective and adequate response to our global security environment. Besides, quantum communications are being considered one of the most promising technologies with applications in security related domains. To this extent, the development of quantum communication infrastructures will significantly impact the architecture of the modern electromagnetic operational environment. Quantum technologies pave the way towards revolutionary technologies by exploiting physical phenomena from different angles and enabling extremely sensitive measurements of multiple parameters including electromagnetic fields. This paper applications for electromagnetic spectrum monitoring and discusses their impact on future electromagnetic warfare operations.
\end{abstract} \\ aims to present a short description of quantum technologies with
}

\section{KEYWORDS:}

Electromagnetic spectrum, quantum communications, quantum sensing, qubits, software defined radio

\section{Introduction}

In the age of big data, the development of new information technologies for the processing and exchange of mass information becomes a major challenge for our society. To meet this growing need, the world of quantum physics offers important possibilities for technological development. The development of quantum physics at the beginning of the $20^{\text {th }}$ century opened the perspective of a completely counter-intuitive subatomic reality. This new perspective paved the way towards modern warfare, where involved actors need to maintain supremacy by controlling state-of-the-art military capabilities including quantum technologies.

Quantum technology is a vast term that is used to refer to different products that make use of the quantum properties of subatomic nature such as quantum 
superposition or quantum entanglement. Briefly, quantum superposition describes how a particle can exist within different states at the same time while quantum entanglement describes the way in which two separate particles can be correlated in such a way that, when one interacts with the other, the other becomes aware and reacts to it (López, 2019). The principles of quantum physics were already applied in various technological devices including lasers, memories of electronic devices, satellite positioning systems and magnetic resonance imaging (MRI) machines.

A series of reports and recommendations were issued to enhance the importance of this emerging field with respect to the promising applications of quantum technologies for defence systems (Till \& Pritchard, 2016; Davies \& Kennedy, 2017; Wolf et al., 2019). Most of the quantum technology being developed or deployed is of dual-use type, meaning that the research effort comes from both military, governmental agencies and private companies or universities. Even if current quantum technologies have mostly low-level Technology Readiness Levels (TRLs) (Krelina, 2021) their potential is extremely promising and developed nations continuously make a sustainable effort in this direction.

The world-wide leader nations in quantum research and development include China, United Kingdom, United States of America, some European nations, and Russia. In 2016, Beijing publicly announced that it urges to achieve major breakthroughs in quantum technologies by 2030 , and that same year it launched the world's first quantum satellite, which teleported a photon to Earth in 2017 (IISS, 2019). In September 2018, the United States published its National Strategic Overview for Quantum Information Science and committed another US $\$ 250 \mathrm{~m}$ to support research grants in the field of quantum sensing, computing and communications (IISS, 2019). Additionally, in October 2020, the United States Department of Defence (DoD) issued an
Electromagnetic Spectrum Superiority Strategy (DoD, 2020) seeking to align electromagnetic spectrum resources, capabilities, and activities across the DoD to support the assumed national security objectives. However, in this recent strategy there is no mentioning of the quantum technologies impact on electromagnetic spectrum superiority while recent published research evoked the potential of using Rydberg atom-based receivers (Halloway et al., 2021). Following, in April 2021, the Defence Advanced Research Project Agency (DARPA) announced a research program that is aimed at enabling the quantum shift in spectrum monitoring (DARPA, 2021). The program goal is to develop radiofrequency (RF) devices that use quantum techniques to access the radio spectrum resources. The objective will be pursued by creating directional RF transceivers with improved sensitivity, bandwidth, and dynamic range.

Although the quantum technology literature is vast, as reported in (Krelina, 2021; Lopez 2019), in the public open literature authors found no study covering the impact of these technologies on spectrum monitoring techniques. To this extent, the aim of this article is to present an in-depth perspective on the possible utilisations of selected quantum technology for improved spectrum sensing capabilities with implications on the outcome of modern military warfare. In the remaining sections of the article, the following taxonomy of quantum technology will be addressed: quantum computing, quantum communications and quantum sensing. The last section of the paper presents the conclusions and highlights the main implications of quantum technologies for future military EMF warfare.

\section{Quantum computing}

To briefly explain the principles employed by quantum computers technologies, it is helpful to clarify how classical computers work. Like all other electronic gadgets, their fundamental units 
of data are bits. This implies their programs and applications are coded in bits, a double dialect that employs $0 \mathrm{~s}$ and $1 \mathrm{~s}$. Every time we connect with one of these devices, such as when touching a key on a keyboard, strings of $0 \mathrm{~s}$ and $1 \mathrm{~s}$ are made, destroyed, or modified in the computer memory.

In classical computing, the bits correspond to circulating electric charges which reflect the passage of an electric current (bit of value 1) or its absence (bit of value 0 ). The reading of the bit is deterministic: 0 or 1 . Likewise, the basic unit of information in quantum computing is the quantum bit, or qubit. From a physical perspective a qubit can be made of any particle with variable energetic excitation states like photons, atoms, electrons, or molecules. The use of qubits makes it possible to represent information by a quantum state which can be the superposition of the states $|0\rangle$ and $|1\rangle$ of the two-level system in variable proportions (DiVincenzo, 2000). The quantum states $|0\rangle$ and $|1\rangle$ of a qubit can represent a state of the lowest respectively the highest particle excitation, while the particle can find itself in any of the infinite intermediate levels of excitation. This represents the fundamental difference between the classical bits and qubits (Figure no. 1). While a classic computer can read only one bit at a time a quantum computer can perform significantly faster as a qubit can be in both states at once.

\section{Bit}

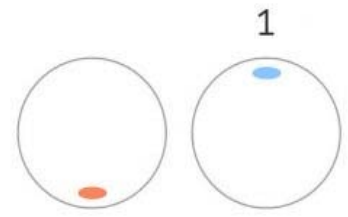

0
Qubit

|1)

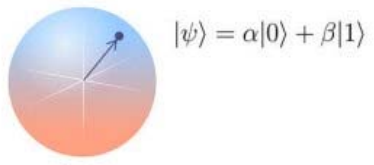

|0)

Figure no. 1: Qubit vs bit. States of classical bit compared to quantum bit superposition (Source: WEB-Dreamstime, 2021)

Physical qubits can be realised by various systems but are most often realised by means of superconducting qubits or trapped-ion qubits. Other technologies include cold atoms, electron spin or photonic qubits but they are still in theory or early development. The use of qubits is limited by several factors but most importantly by their quality and coherence time. Because of the limited fidelity obtained by using solely physical qubits, efficient communications need to enable complex error correction codes. This gave birth to logical qubits consisting of multiple physical qubits and a set of error correction codes. Quantum computers use a process called quantum parallelism enabling more efficient algorithms that can exponentially outperform classical computers. That is, with only $n$ qubits, we can obtain a computing capacity equivalent to $2^{\mathrm{n}}$ bits (Lopez, 2019).

In the race for quantum computers, the start was given in 1998 by a 2-qubit computer built by scientists at the Oxford University, IBM, Berkley, Stanford, and MIT. The following couple of decades brought little achievements for increasing the number of qubits that quantum computers could contain but in 2017 IBM company was the first to develop a 17-qubit followed by a 50 -qubit quantum computer (Feldman, 2019). In a recent press release IBM announced a 433-qubit quantum processor in 2022 and 1,000 qubits by 2023 
(Gambeta, 2020). Figure no. 2 presents the progresses in the development of quantum computers in terms of number of physical qubits.

The race for quantum computers is yet to come as the leap consists of implementing logical qubits rather that physical ones. According to some experts, the number of physical qubits needed to obtain a logical one could reach up to one thousand (López 2019). Roetteler et al. (2017) appreciate that a quantum computer with at least 1500 logical qubits will probably have the capacity to break all existing cyber security.

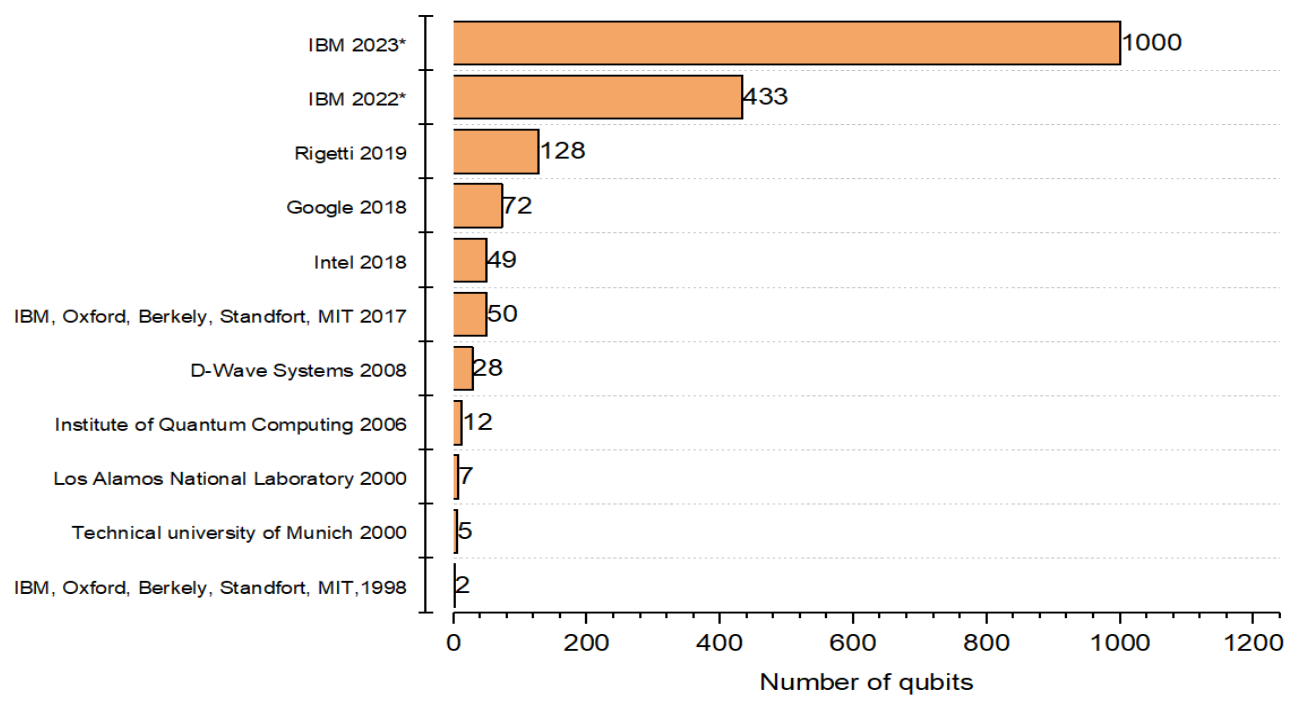

Figure no. 2: The evolution of number of physical qubits in quantum computers (Source: Authors)

Software defined radio (SDR) a computer connected to the SDR device via technology is already employed for spectrum monitoring purposes and has proven its agility for deploying real-time electromagnetic field (EMF) monitoring systems (Llames, 2016; Ball, 2017; Helbet, 2021). A SDR system is based on a peripheral hardware component connected to a personal computer. Traditional hardware components like mixers, filters or amplifiers are instead implemented by means of software on the personal computer. A basic SDR EMF measurement system consists of an USB/serial/Ethernet/SFP/SFP+ interface. The SDR devices currently available on the market can use up to 4 synchronised reception channels but low-cost devices usually have only one reception channel. Figure no. 3 presents the current architecture of a SDR system built for EMF measurements. When powerful quantum computers will be available, software defined radio capabilities will also improve as they play an important role in the system's performance.
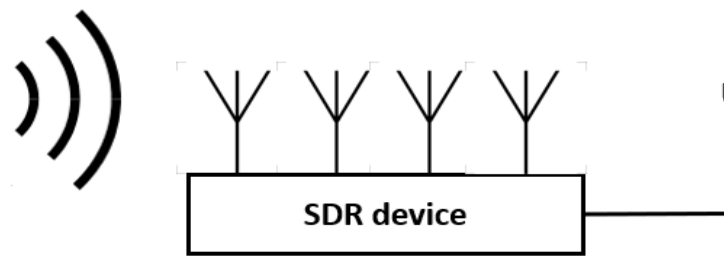

USB/Serial/Ethernet/SFP+ conectivity

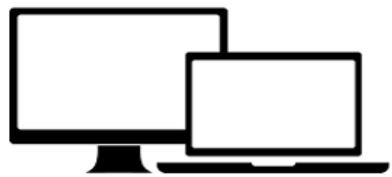

Figure no. 3: SDR system architecture for EMF measurements

(Source: Authors) 
Several quantum start-ups are developing hybrid software solutions between supercomputers and quantum computers. This type of hybrid computers could provide the analytical power needed to compute, in real-time, a wider range of the electromagnetic spectrum and thus improve EMF spectrum monitoring by SDRs.

\section{Quantum communications}

Quantum communication is a term used to describe data exchange by means of an entirely quantum network that uses optical fibre or free space channels as a transmission medium. Traditionally, this type of communication uses a photon as an information carrier. By allowing the distribution of entangled states, quantum communications can have several promising applications inaccessible with current technologies: long-distance interferometry, time reference for atomic clock synchronization, quantum computer networking, quantum key distribution, etc. Remote access to quantum computers and the sharing of computations between several quantum computers makes it possible to associate several processors to increase their capacities exponentially. The practical use of quantum communications will be based on the ability to develop quantum photonic components that can be integrated into existing communications infrastructures without requiring specific deployments.

Chinese researchers were the first to set up a quantum communication network, combining over 700 optical fibres on the ground with two ground-to-satellite links. Their network employed quantum key distribution over a total distance of 4,600 kilometres for users across the country (Chen, 2021). An outline of the network architecture is visible in Figure no. 4.

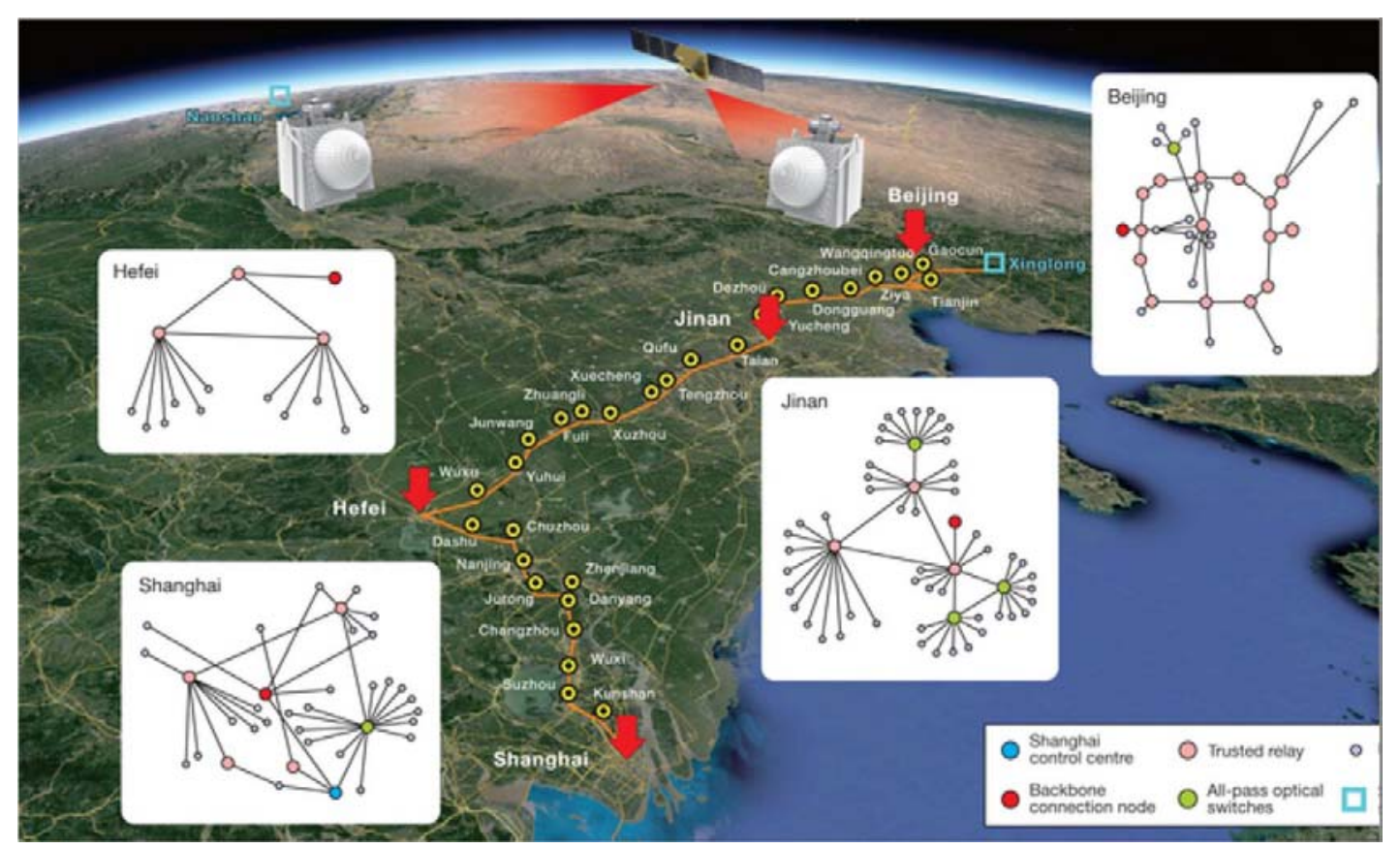

Figure no. 4: Integrated quantum communication network

(Source: University of Science and Technology of China, 2021)

Based on a similar network topology we can deploy an EMF measurement network based on quantum communications. In Figure no. 5 we present such a network design for Romania. The EMF measurement sensor could be traditional or even better can be replaced by quantum sensors. High-capacity communication channels are 
needed as today's sensors can deliver high resolution mapping of the EMF spectrum in real time. To this extent, the use of quantum technology assures both capacity and security requirements for both ground and satellite to ground communication links.

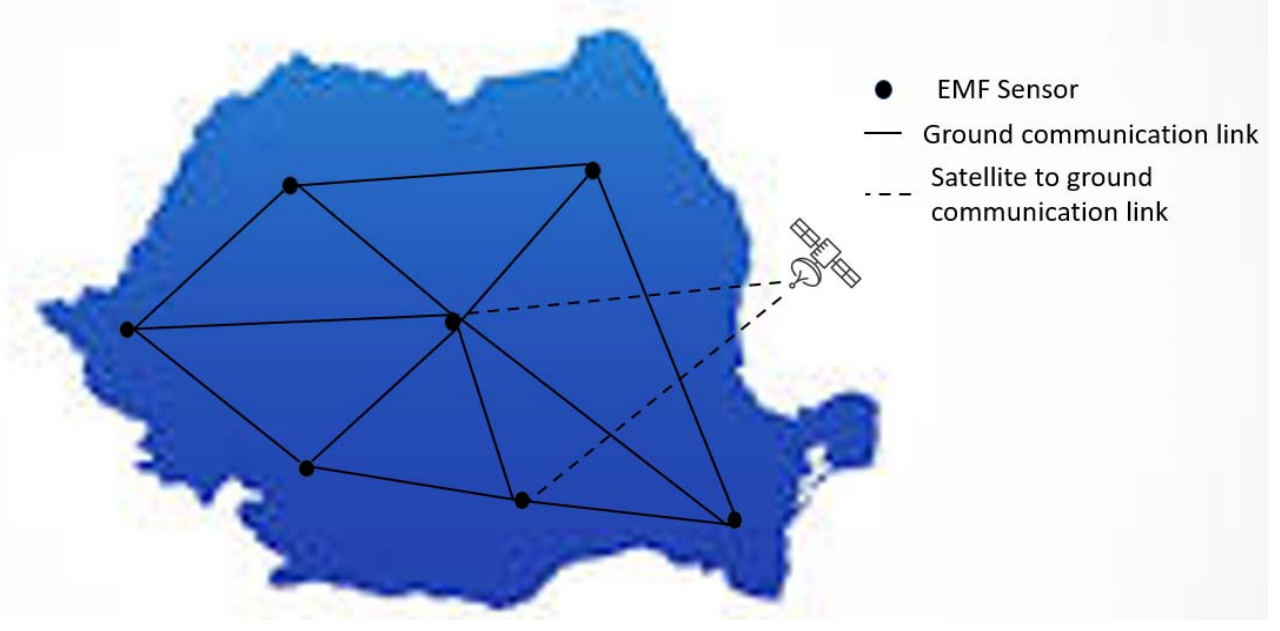

Figure no. 5: EMF measurement network based on quantum communication (Source: Authors)

For satellite to ground communication high-capacity laser communication (Lasercom) can be enabled. Lasercom development was accelerated by the rapid growth of satelliteconstellation programmes used for either remote sensing or communications. The use of quantum communications for lasercom conducts to improved performances and represents a prerequisite for the most promising cryptography technique known as quantum key distribution (QKD). The first milestone for practical implementation of QKD was achieved by scientist at the Los Alamos National Laboratory (Hiskett et al., 2006) and University of Cambridge (Dixon et al., 2008) in 2007 and 2008 respectively. The efforts were continued by researchers at the University of Geneva in 2015, when they experimentally proved QKD over a $307 \mathrm{~km}$ optical fibre (Korzh et al., 2015). Today, China is the main leader in QKD advances - as it first demonstrated the use of QKD over a satellite to ground communication link (Yin, 2020). With the development of free space communication over optical link, techniques are needed for developing adequate interception and control mechanisms. The quantum shift will enable the use of quantum eavesdropper as third-party communication entity. Even if not always looked at from this perspective, light is a high frequency electromagnetic wave. With traditional radiofrequency devices migrating to higher frequencies and the spread of optical communication links one must reconsider the traditional electromagnetic spectrum monitoring methods. To this extent both sensors and measurement instrument response must be adapted for the new requirements.

\section{Quantum sensing}

Quantum sensing stands for the use of a quantum system, quantum properties, or quantum phenomena to perform a measurement of a physical quantity such as electric and magnetic field strengths.

If many of the quantum technology applications are rather theoretical and exhibit low TRLs, with long-term time expectation, the field of quantum sensing is the most mature quantum technology domain presently. The most representative technological example for quantum sensing 
devices is probably atomic clocks, which have been around for almost 50 years as part of the Global Positioning System (GPS).

In the case of civilian applications (smart homes, self-driving cars, traffic control, industry, medicine, etc.), quantum sensors find themselves in direct competition with more conventional sensors whose performance continues to improve. However, they are called upon to play a leading role in the fields of defence applications: navigation, electromagnetic spectrum monitoring and remote sensing. From this perspective we may recall:

- Quantum navigation sensors (based on atoms cooled by laser or on impurities in diamonds) are capable of precisely measuring variations in certain physical properties of the terrestrial globe such as electromagnetic or gravitational fields. High-definition cartography makes it possible to position oneself without having to resort to GPS.

- Electromagnetic sensing could be much more efficient by using sensors based on impurities in diamond to perform spectral analysis of electromagnetic signals several orders of magnitude finer than current technologies.

- Quantum radar is an emerging remote sensing technology based on quantum illumination. If it keeps its promises, it will be able to detect stealth planes, filter out deliberate jamming attempts, etc.

In Thales laboratories, numerous studies are carried out on superconducting quantum interference devices for the design and production of miniature quantum antennas, covering a very wide spectrum of waves, usable in brain imaging or in particle detection. Solid-state quantum sensors are being researched to demonstrate their ability to measure very weak magnetic fields (for magnetic resonance imaging MRI, for example). Rare ions will allow the analysis of a radio frequency spectrum over broad spectral bands continuously aimed at de-cluttering and optimizing networks. Other themes are being developed, such as sensors for future quantum inertial units on board aircraft (Damiano, 2021).

The most promising quantum technique for EMF spectrum monitoring is represented by employing the principle of electromagnetically induced transparency of Rydberg atoms. A quantum-based antenna was developed and proved its feasibility for spectrum sensing over wider broadband RF carrier (Song et al., 2019).

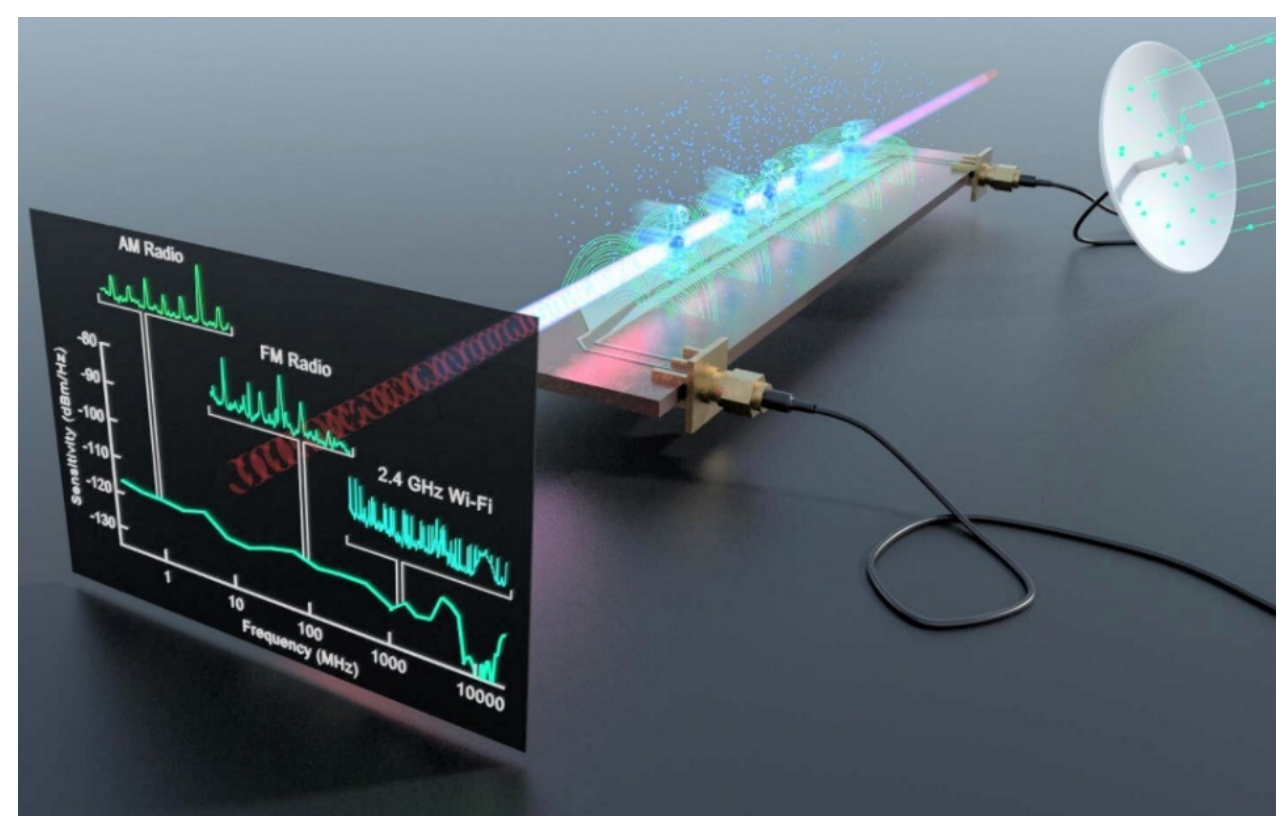

Figure no. 6: Rydberg receiver detecting a wide range of signals

(Source: US Army illustration, US Army DEVCOM, 2021) 
US Army scientists have designed a quantum sensor capable of measuring RF spectrum from 0 to $20 \mathrm{GHz}$. This EMF spectrum analyser is expected to surpass fundamental limitations of traditional electronics in sensitivity, bandwidth, and frequency range (Meyer et al., 2021). Following, DARPA has launched a 56-month research program for enabling the quantum shift in electromagnetic spectrum monitoring.

Other technological products based on quantum physics that were developed for EMF spectrum sensing purposes are electro-optic probes. In recent years, great
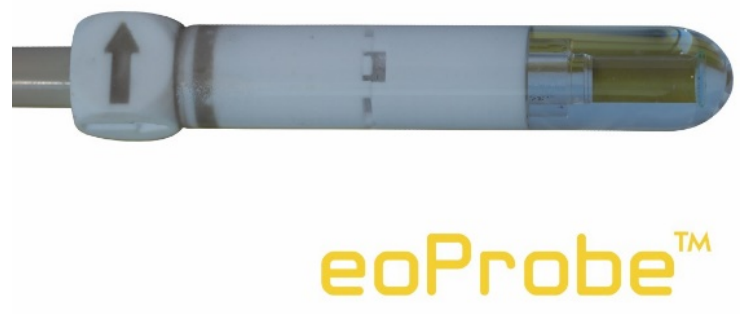

Figure no. 7: Electric field sensors - electro-optic type from

(a) Kapteos (France)

\section{Conclusions}

This article presents a previsioned impact of some selected quantum technologies on EMF measurement systems. The technologies discussed include quantum computers, quantum networks and quantum sensing.

The ongoing research in the field of quantum communication networks is based on extremely precise optical links. The flexibility and open-source character of SDR applications recommends them for integration into hybrid electro-optic architecture of next generation networks. With SDRs already having optical interfaces like SFP+ we can expect to see them in this type of networks as soon as they are being deployed. Moreover, with the development of quantum/ hybrid processors, on the long term, we expect to see traditional EMF measurement progress has been made in the optical sensing technique for EMF measurements that include the development of magnetic and electric field optical sensors based on Faraday effect, magneto-strictive materials, electric-optic crystals, and piezoelectric materials (Peng et al., 2019). The use of electro-optic field probes has a series of advantages as size and weight, immunity to EMF, high sensitivity or wide dynamic range. In Figure no. 7 we show two commercially available electro-optic field probes from Kapteos (a) and Seikoh-Giken (b).

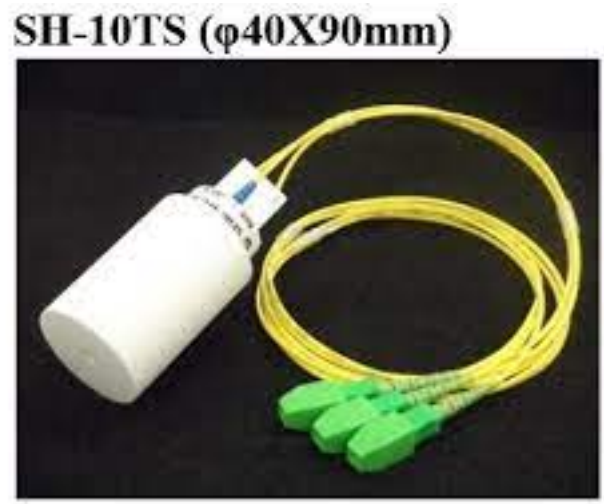

devices equipped with this type of technology. This increase in processing capacity would lead to real-time analysis of wider portions of the EMF spectrum.

Among all the analysed quantum technologies the authors believe that the most significant impact in the following few years will come from quantum-sensors based on Rydberg atoms behaviour. The TRL level of the technology is rather high compared to other quantum technologies discussed in this article (eg. High performance quantum computers) and ongoing research is being conducted in this field. Research in this area will contribute to defence system's modernization for the next-generation computer networks by introducing novel communications techniques of RF signal detection. In the last two years significant progress has been made to the 
field by running Rydberg atomic sensor over a wide frequency range. Scientists are expected to find means to boost sensitivity as well as expand detection techniques to include more complicated waveforms. The developed sensor is small sized and virtually undetectable, characteristics that recommend it for usage in combat areas.

As pointed out in the previous sections, some applications of quantum technologies offer a wide range of applications in modern electromagnetic spectrum (EMS) warfare. This new technology is expected to improve sensitivity, efficiency, introduce new capabilities, and sharpen present military technologies used for EMS supremacy. To this extent, electromagnetic spectrum superiority is a precursor to the successful conduct of operations in all operational environments (land, air, sea, space, cyberspace). In other to assure their supremacy, nations are required to ensure superiority by combining efforts to improve technological capabilities, activities, and operations in the short and long term. As pointed out by the example of powerful nations, the research in the field of quantum technologies will enable a paradigm shifting that can assure both technological and military supremacy.

\section{REFERENCES}

Ball, D., Naik, N., \& Jenkins, P. (2017). Spectrum Alerting System Based on Software Defined Radio and Raspberry Pi. Sensor Signal Processing for Defence Conference (SSPD), 1-5, DOI: 10.1109/SSPD.2017.8233266.

Chen, Y.A., Zhang, Q., Chen, T.Y. et al. (2021). An integrated space-to-ground quantum communication network over 4,600 kilometres. Nature, Vol. 589, 214-219, https://doi.org/10.1038/s41586-020-03093-8

Damiano, J.-P.e. (2021). Les technologies quantiques. Contexte et enjeux, applications et perspective. IESF Côte d'Azur, Bull. Nr. 2.

Davies, A., \& Kennedy, P. (2017). Special report - From little things: Quantum technologies and their application to defence. ASPI (Australian Strategic Policy Institute).

Defense Advanced Research Projects Agency (DARPA). (2021). DARPA Selects Research Teams to Enable Quantum Shift in Spectrum Sensing. Available at: https://www.darpa.mil/news-events/2021-08-04.

Department of Defense. (2020). Electromagnetic Spectrum Superiority Strategy.

Di Vincenzo, D.P. (2000). The Physical Implementation of Quantum Computation. Fortschritte der Physik, Vol. 48, 771.

Dixon, A.R., Yuan, Z.L., Dynes, J.F., Sharpe, A.W., \& Shields, A.J. (2008). Gigahertz decoy quantum key distribution with $1 \mathrm{Mbit} / \mathrm{s}$ secure key rate. Optics Express. Vol. 16, Issue 23, 18790-7. arXiv:0810.1069. Bibcode:2008OExpr..1618790D. DOI:10.1364/OE.16.018790. PMID 19581967. S2CID 17141431

Dreamstime. (2021). Available at: https://www.dreamstime.com/qubit-vs-bit-statesclassical-compare-to-quantum-superposition-vector-eps-image199368498, accessed on 15 November 2021.

Feldman, S. (2019). 20 years of quantum computing growth. Available at: https:/www.statista.com/chart/17896/quantum-computing-developments/.

Gambetta, J. (2020). IBM's Roadmap for Scaling Quantum Technology. IBM, available at: https://www.ibm.com/blogs/research/2020/09/ibm-quantum-roadmap/ 
Helbet, R., Bechet, P., Monda, V., Miclăuş, S., \& Bouleanu, I. (2021). Low-Cost Sensor Based on SDR Platforms for TETRA Signals Monitoring. Sensors, Vol. 21, 3160. https://doi.org/10.3390/s21093160

Hiskett, P.A., Rosenberg, D., Peterson, C.G., Hughes, R.J., Nam, S., Lita, A.E., Miller, A.J., \& Nordholt, J.E. (2006). Long-distance quantum key distribution in optical fibre. New Journal of Physics. IOP Publishing, Vol. 8, Issue 9, 193. arXiv:quant-ph/0607177. Bibcode:2006NJPh...8..193H. DOI:10.1088/1367-2630/8/9/193. ISSN 1367-2630.

Holloway, C., Simons, M., Haddab, A.H., Gordon, J.A., Anderson, D.A., Raithel, G., \& Voran, S. (2021). A Multiple-Band Rydberg Atom-Based Receiver: AM/FM Stereo Reception. IEEE Antennas and Propagation Magazine, Vol. 63, Issue 3, 63-76. https://doi.org/10.1109/map.2020.2976914

IIIS, The Military Balance. (2019). Available at: https://www.iiss.org/publications/themilitary-balance/the-military-balance-2019/quantum-computing-and-defence

Korzh, B., Lim, Ch.C.W., Houlmann, R., Gisin, N., Li, M.J., Nolan, D., Sanguinetti, B., Thew, R., \& Zbinden, H. (2015). Provably Secure and Practical Quantum Key Distribution over $307 \mathrm{~km}$ of Optical Fibre. Nature Photonics, Vol. 9, Issue 3, 163-168. arXiv: 1407.7427. Bibcode: 2015NaPho...9..163K. DOI:10.1038/nphoton.2014.327. S2CID 59028718

Krelina, M. (2021). Quantum Warfare: Definitions, Overview and Challenges. Available at: https://arxiv.org/abs/2103.12548

Llames, G.J.M., \& Banacia, A.S. (2016). Spectrum Sensing System in Software-defined Radio to Determine Spectrum Availability. IEIE Transactions on Smart Processing and Computing, Vol. 5, Issue, 2, 100-106. https://doi.org/10.5573/ieiespc.2016.5.2.100.

López, M.A. (2019). Quantum Technologies - Digital transformation, social impact, and cross sector disruption, IDB.

Meyer, D.H., Kunz, P.D., \& Cox, K.C. (2021). Waveguide-Coupled Rydberg Spectrum Analyzer from 0 to $20 \mathrm{GHz}$. Physical Reviews Applied, Vol. 15, Issue 1 . https://doi.org/10.1103/physrevapplied.15.014053

Peng, J., Jia, S., Bian, J., Zhang, S., Liu, J., Zhou, X. (2019). Recent Progress on Electromagnetic Field Measurement Based on Optical Sensors. Sensors. Vol. 19, Issue 13, 2860. https://doi.org/10.3390/s19132860

Roetteler, M., Naehrig, M., Svore, K.M, \& Lauter, K. (2017). Quantum resource estimates for computing elliptic curve discrete logarithms. Advances in Cryptology ASIACRYPT 2017: 23 ${ }^{\text {rd }}$ International Conference on the Theory and Applications of Cryptology and Information Security, Hong Kong, China, December 3-7, Proceedings, Part II (241-270).

Song, Z., Liu, H., Liu, X., Zhang, W., Zou, H., Zhang, J., \& Qu, J. (2019). Rydbergatom-based digital communication using a continuously tunable radio-frequency carrier. Optics Express, Vol. 27, Issue 6, 8848. https://doi.org/10.1364/oe.27.008848

Till, S., \& Pritchard, J. (2016). UK quantum technology landscape 2016. DSTL/PUB098369. UK National Quantum Technologies Programme.

U.S. Army DEVCOM. (2021). Army researchers detect broadest frequencies ever with novel quantum receiver. Available at: https://www.army.mil/article/242980/army researchers detect broadest frequencies ever with quantum receiver

Wolf, Stuart A. et al. (2019). Overview of the Status of Quantum Science and Technology and Recommen-dations for the DoD. Institute for Defense Analyses.

Yin, J., Li, Y.H., Liao, SK. et al. (2020). Entanglement-based secure quantum cryptography over 1,120 kilometres. Nature, 582, 501-505, available at: https://doi.org/10.1038/s41586-020-2401-y 